Helium ion microscopy of graphene: beam damage, image quality and edge contrast

This content has been downloaded from IOPscience. Please scroll down to see the full text. 2013 Nanotechnology 24335702

(http://iopscience.iop.org/0957-4484/24/33/335702)

View the table of contents for this issue, or go to the journal homepage for more

Download details:

IP Address: 134.226.8.83

This content was downloaded on 07/10/2014 at 13:45

Please note that terms and conditions apply. 


\title{
Helium ion microscopy of graphene: beam damage, image quality and edge contrast
}

\author{
D Fox ${ }^{1}$, Y B Zhou ${ }^{1}$, A O’Neill ${ }^{1}$, S Kumar ${ }^{2}$, J J Wang ${ }^{1}$, J N Coleman ${ }^{1}$, \\ G S Duesberg ${ }^{2}$, J F Donegan ${ }^{1}$ and $\mathrm{H} \mathrm{Z} \mathrm{ZZ^{2 } \text { Dang } ^ { 1 }}$ \\ ${ }^{1}$ School of Physics and CRANN, Trinity College Dublin, Dublin 2, Republic of Ireland \\ ${ }^{2}$ School of Chemistry and CRANN, Trinity College Dublin, Dublin 2, Republic of Ireland \\ E-mail: Hongzhou.Zhang@tcd.ie
}

Received 20 April 2013, in final form 11 June 2013

Published 24 July 2013

Online at stacks.iop.org/Nano/24/335702

\begin{abstract}
A study to analyse beam damage, image quality and edge contrast in the helium ion microscope (HIM) has been undertaken. The sample investigated was graphene. Raman spectroscopy was used to quantify the disorder that can be introduced into the graphene as a function of helium ion dose. The effects of the dose on both freestanding and supported graphene were compared. These doses were then correlated directly to image quality by imaging graphene flakes at high magnification. It was found that a high magnification image with a good signal to noise ratio will introduce very significant sample damage. A safe imaging dose of the order of $10^{13} \mathrm{He}^{+} \mathrm{cm}^{-2}$ was established, with both graphene samples becoming highly defective at doses over $5 \times 10^{14} \mathrm{He}^{+} \mathrm{cm}^{-2}$.

The edge contrast of a freestanding graphene flake imaged in the HIM was then compared with the contrast of the same flake observed in a scanning electron microscope and a transmission electron microscope. Very strong edge sensitivity was observed in the HIM. This enhanced edge sensitivity over the other techniques investigated makes the HIM a powerful nanoscale dimensional metrology tool, with the capability of both fabricating and imaging features with sub-nanometre resolution.
\end{abstract}

S Online supplementary data available from stacks.iop.org/Nano/24/335702/mmedia

(Some figures may appear in colour only in the online journal)

\section{Introduction}

The helium ion microscope (HIM) is a recently developed scanning ion microscope [1]. Images acquired in the HIM are formed by detecting secondary electrons generated within the sample by the primary helium ion beam. At first glance these images often appear similar to those produced in the scanning electron microscope (SEM). However, many differences exist between these two imaging techniques as a direct result of the different beam-sample interactions occurring. At the typical acceleration voltage of $30 \mathrm{kV}$ the HIM produces secondary electrons (SE) with a very low energy distribution [2]. These electrons can only escape from the top few nanometres of a sample [3], resulting in very surface sensitive imaging [4].
The helium ion beam remains well collimated within the SE generation depth [5], and the backscattered helium ion yield is generally very low [6]. This results in the highest resolution type of SEs (SE1 electrons) being the main signal produced by the helium ion beam [7]. The HIM has been used to acquire the highest resolution SE imaging of a bulk specimen to date [8]. The HIM also has enhanced topographical contrast and enhanced material contrast over SEM imaging [9]. A backscattered ion imaging mode can provide complementary information such as channelling contrast [10] and material contrast from sub-surface particles [11].

The strong and highly localized interaction of the helium ions in a sample make the HIM a powerful tool for applications such as critical dimension measurements [12, 13] 
and also, at increased doses, direct milling of nanoscale features. Ohya et al simulated the edge contrast of silicon nanostructures observed in HIM and SEM [14]. They concluded that HIM imaging would continue to show strong edge contrast, even down to sub-nanometre feature sizes. This is due to the stronger dependence of SE yield on the helium ion incidence angle than the electron incidence angle in the SEM. Postek et al experimentally found that the HIM showed greater edge contrast of amorphous silicon lines than the SEM at equivalent acceleration voltages [15]. While the edge contrast could be enhanced in SEM by lowering the acceleration voltage this comes at the expense of reducing the imaging resolution. The HIM produces images with a greater signal to noise ratio (SNR) as the imaging dose is increased. However, the higher dose was found to lead to material removal, reducing the size of the features that were being measured [14]. Imaging with sub-nanometre resolution in the HIM comes at the inherent cost of locally modifying the sample.

Graphene [16], one of the most interesting and widely researched materials in recent years [17-20], has been confined into $5 \mathrm{~nm}$ wide ribbons by HIM milling [21]. This confinement can open an electronic bandgap, something graphene requires for switching operations [22]. However, the crystal structure of the graphene must remain intact during the HIM processing of the graphene. In our previous work the effect of HIM irradiation on silicon has been investigated [23] and the effects of electron beam and argon ion irradiation on graphene have been evaluated [24, 25]. The HIM beam induced damage and amorphization of graphene have yet to be comprehensively investigated.

In this work we investigate the effect of a range of HIM irradiation doses on the lattice structure of both freestanding and supported graphene by Raman spectroscopy. Raman spectroscopy can probe the vibrational modes of a crystal structure. It is a rapid, non-destructive characterization tool which is widely used in the analysis of graphene. It is also very sensitive to the structure of crystals and in the case of graphene it can be used to detect the density of defects, the sample thickness and any doping which may have occurred.

We then correlate these irradiation doses with the corresponding HIM image quality in order to find the signal to noise ratio which can be achieved at a non-destructive imaging dose. We acquire HIM, SEM and transmission electron microscope (TEM) images of a single graphene flake for comparison of the edge contrast these imaging techniques produce.

\section{Experimental details}

Our freestanding graphene sample was prepared by the chemical vapour deposition (CVD) method. A $1 \mathrm{~cm} \times 1 \mathrm{~cm}$ copper foil was placed in a furnace and heated to $1000^{\circ} \mathrm{C}$ in vacuum. Methane and hydrogen were flowed through the furnace causing carbon to deposit on the copper surface. The furnace was then cooled, leaving a single, continuous layer of graphene on the copper film. The graphene was transferred to a Si substrate with arrays of $2 \mu \mathrm{m}$ holes by the following steps. A $200 \mathrm{~nm}$ polymer (PMMA) layer was spin coated onto the sample. The copper foil was etched away with $\mathrm{FeCl}_{3}$. The graphene and polymer layers were transferred to a Si substrate. The polymer layer was removed in hot acetone. The sample was then transferred into hot isopropanol, while remaining in liquid at all times. Finally, the sample was taken out of the isopropanol slowly and left to dry in air. Some residual polymer contaminants remain after this process [26]. The effect of this contamination has been observed to introduce some disorder in the graphene [27, 28]. The supported graphene sample was prepared by the same process as above except that the graphene was transferred onto a $300 \mathrm{~nm} \mathrm{SiO}_{2}$ layer grown on a $\mathrm{Si}$ substrate. Single layers were identified by optical contrast.

An Orion Plus helium ion microscope operating at an acceleration voltage of $30 \mathrm{kV}$ was used to irradiate both graphene samples. Seven areas on each sample were irradiated with doses of $10^{13}, 5 \times 10^{13}, 10^{14}, 5 \times 10^{14}, 10^{15}, 5 \times 10^{15}$ and $10^{16} \mathrm{He}^{+} \mathrm{cm}^{-2}$. This exposure was achieved by using the internal patterning software to raster the focused helium beam over a $2 \mu \mathrm{m} \times 2 \mu \mathrm{m}$ area with a $5 \mathrm{~nm}$ pixel spacing. A beam current of $1 \mathrm{pA}$ was used and the dwell time per pixel was adjusted to achieve the required dose.

Raman spectra were acquired from the irradiated areas using a $633 \mathrm{~nm}$ HeNe laser with a Horiba Jobin Yvon LabRAM-HR. A $100 \times$ objective lens and a diffraction grating with 1200 lines $\mathrm{mm}^{-1}$ were used. The spatial resolution of the laser was $\sim 0.7 \mu \mathrm{m}$. A $10 \mathrm{~s}$ acquisition time and four spectrum averages were used at each point. The most prominent features of the Raman spectrum of graphene are the characteristic $G$ peak at $\sim 1580 \mathrm{~cm}^{-1}$ and the $2 \mathrm{D}$ peak at $\sim 2700 \mathrm{~cm}^{-1}$. A third peak, the $D$ peak at $\sim 1350 \mathrm{~nm}^{-1}$, becomes Raman active in defective graphene. In the low defect density regime the ratio of the $\mathrm{D}$ peak intensity to the $\mathrm{G}$ peak intensity $\left(I_{\mathrm{D}} / I_{\mathrm{G}}\right)$ is proportional to the defect density [29].

Graphene flakes were prepared by sonicating pristine graphite in isopropanol for $48 \mathrm{~h}$. After sonication, the sample was centrifuged at $2000 \mathrm{rpm}$ for $45 \mathrm{~min}$ to remove large chunks of graphite that did not fully exfoliate. The resulting supernatant was decanted and retained for further use. The dispersion quality was similar to that previously reported by O'Neill et al [30] with flakes typically $1 \mu \mathrm{m}$ in length and with thicknesses of less than ten layers. A couple of drops of the solution were dropped directly onto a holey carbon support film on a TEM grid.

In order to correlate the ion doses analysed by Raman spectroscopy with image quality in the HIM a graphene flake was selected in the HIM. Flakes were used for this part of the project in order to obtain good edge contrast. The graphene flake was suspended above a carbon coated hole which is several millimetres deep. This set up produces minimal background noise in the images from secondary electrons and backscattered ions produced beneath the graphene sample. The flake was imaged with a field of view of $500 \mathrm{~nm}$. This is approximately the minimum magnification at which the sub-nanometre resolution of the tool can still be demonstrated. Higher magnifications would make it impossible to image with the extremely low dose we require. Even at this 

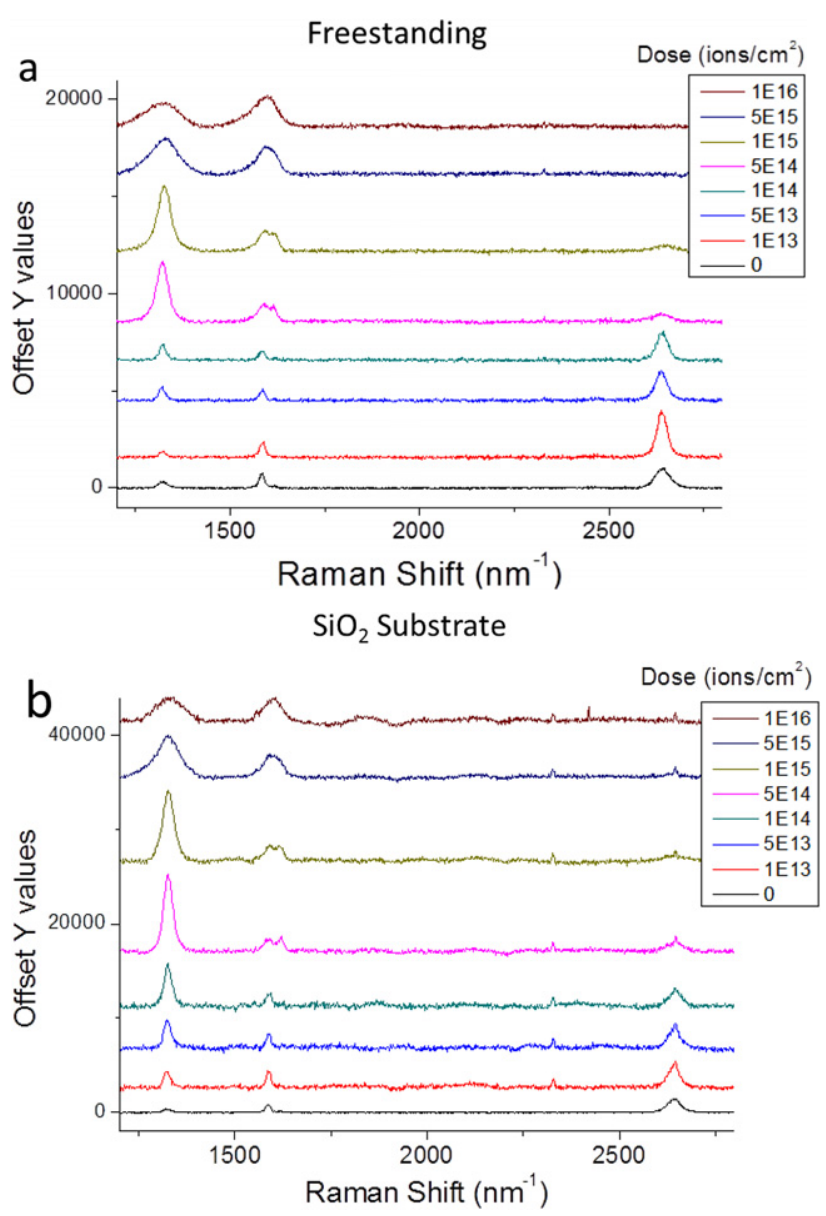

Figure 1. Raman spectra of freestanding (a) and supported (b) graphene samples irradiated with the various doses shown. In both cases the dose increases from the bottom spectrum up to the top spectrum. The D peak at $\sim 1350 \mathrm{~nm}^{-1}$ and the $G$ peak at $\sim 1580 \mathrm{~nm}^{-1}$ are the peaks which were used to plot the $I_{\mathrm{D}} / I_{\mathrm{G}}$ ratios in figure 2 .

magnification an image with a dose of $10^{13} \mathrm{He}^{+} \mathrm{cm}^{-2}$ could not be acquired as our beam blanker could not blank at a fast enough rate to acquire this image with the parameters used. Images of the flake were acquired with doses of $5.2 \times$ $10^{13}, 1.3 \times 10^{14}, 5.2 \times 10^{14} \mathrm{He}^{+} \mathrm{cm}^{-2}$. These images were acquired with a beam current of $0.4 \mathrm{pA}$, a field of view of $500 \mathrm{~nm}$ and a resolution of $512 \times 512$ pixels. The dwell times used were $0.2,0.5$ and $2 \mu$ s respectively. A final image was acquired with a much higher dose of $1.3 \times 10^{17} \mathrm{He}^{+} \mathrm{cm}^{-2}$. This is the typical dose used when acquiring a resolution image from which the sub-nanometre probe size of the HIM can be measured. For this image the dwell time was $1 \mu \mathrm{s}$, the resolution was increased to $2048 \times 2048$ and 32 line averages were used.

Another graphene flake was identified on the TEM grid used above. An image of this flake was acquired with a Carl Zeiss Ultra Plus SEM using the in-lens detector. The microscope was operating at a $20 \mathrm{kV}$ acceleration voltage. A $2.6 \mathrm{~mm}$ working distance was used. The same flake was then imaged in an FEI Titan 80-300 TEM operating at $300 \mathrm{kV}$. Scanning-TEM (STEM) mode was used with a high-angle annular dark-field (HAADF) detector to acquire the image.

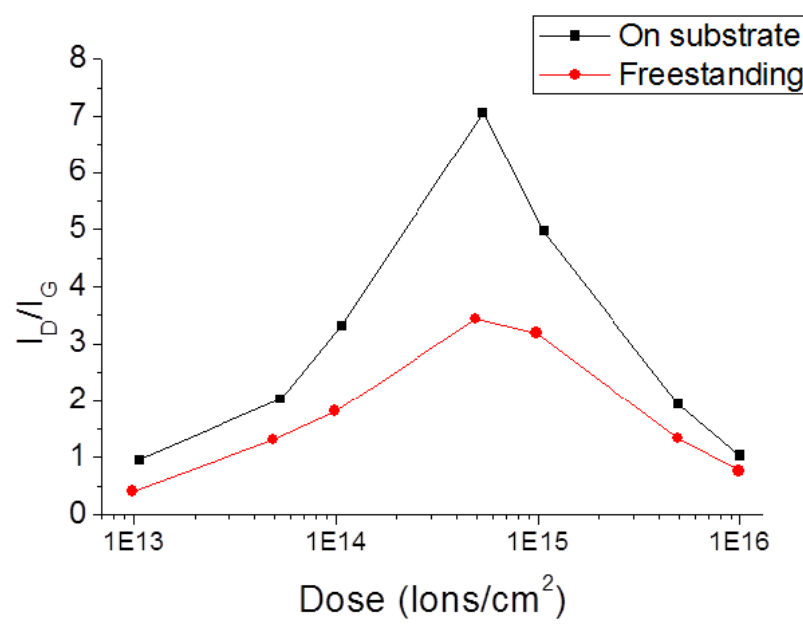

Figure 2. The graphene Raman spectrum $D-G$ peak intensity ratio plotted as a function of the log of the ion irradiation dose. A higher D-G peak intensity ratio indicates a higher defect density; however this is only the case within a low defect density regime. Above $5 \times 10^{14} \mathrm{He}^{+} \mathrm{cm}^{-2}$ both samples become highly defective and no longer follow this rule. The freestanding graphene sample consistently exhibits a lower density of defects.

Finally the flake was imaged in the HIM, which was operating at $30 \mathrm{kV}$. A very low beam current of $0.4 \mathrm{pA}$ was used in the HIM to minimize sample damage.

\section{Results and discussion}

Seven Raman spectra from the seven irradiated areas on the freestanding and supported graphene samples were acquired. These spectra are shown for freestanding and supported graphene in figures 1 (a) and (b) respectively. The $I_{\mathrm{D}} / I_{\mathrm{G}}$ ratios for each spectrum were found and are plotted as a function of the log of the irradiation dose in figure 2. It is clear from the first four data points in each graph that the supported graphene sample consistently has a greater defect density (higher $I_{\mathrm{D}} / I_{\mathrm{G}}$ ratio) than the freestanding graphene sample, at the same irradiation dose. At a dose above $5 \times 10^{14} \mathrm{He}^{+} \mathrm{cm}^{-2}$ the $I_{\mathrm{D}} / I_{\mathrm{G}}$ ratio can no longer be used as an indicator of the defect density in the sample [31]. Above this dose the peaks are observed to become progressively broader. Also the $2 \mathrm{D}$ peak is not present in the highest dose samples. At this point the material has become heavily defective.

The supported graphene sample experiences more damage than the freestanding graphene sample for several reasons. Stopping and range of ions in matter (SRIM) is a widely used and freely available software package [32]. SRIM uses Monte Carlo modelling to plot the trajectories and collision details of ions travelling through a sample. The ion species and energy and the sample composition are configurable. We used a SRIM simulation to find the backscatter yield of helium from the sample. Backscattered ions can interact a second time with the graphene layer on the surface. The sample was set up as a $0.34 \mathrm{~nm}$ carbon layer on a $300 \mathrm{~nm} \mathrm{SiO}_{2}$ layer on a $\mathrm{Si}$ substrate $(\mathrm{C}$ density $=$ $2.25 \mathrm{~g} \mathrm{~cm}^{-3}, \mathrm{SiO}_{2}$ density $\left.=2.65 \mathrm{~g} \mathrm{~cm}^{-3}\right)$. The trajectories of $1000030 \mathrm{keV}$ helium ions in the sample were simulated. 


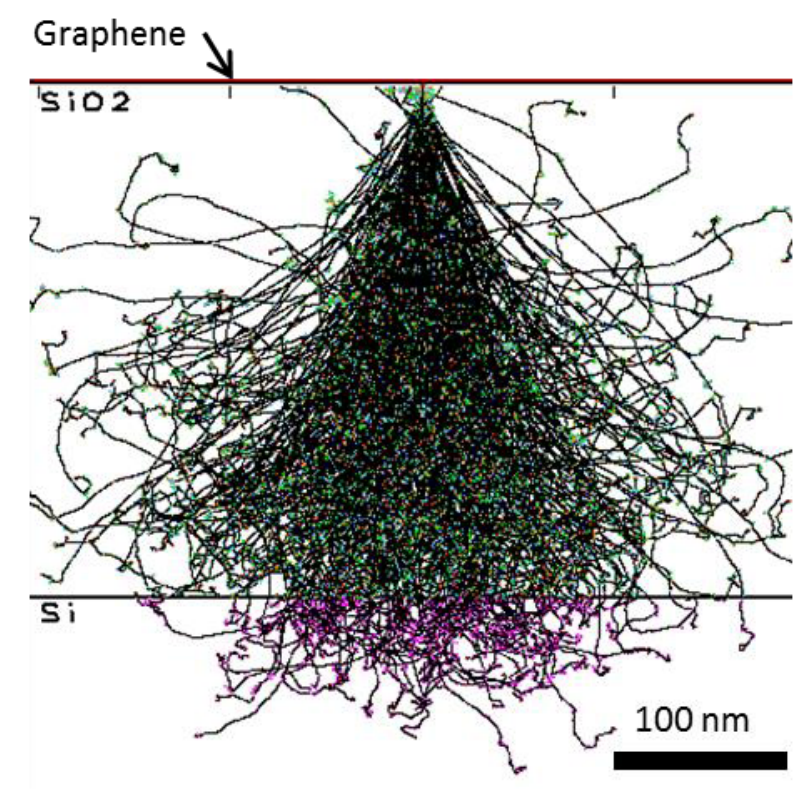

Figure 3. SRIM simulation of the trajectory of $30 \mathrm{keV}$ helium ions. The sample is single layer graphene on $300 \mathrm{~nm} \mathrm{SiO}_{2}$ on $\mathrm{Si}$. 500 ion trajectories are shown. 10000 ion trajectories were simulated to obtain a value for the yield of backscattered helium ions. $0.94 \%$ of the ions were backscattered. Sputtering yields of 0.035 for C, 0.003 for $\mathrm{Si}$ and 0.005 for $\mathrm{O}$ were also calculated from the simulation.

The damage calculation mode was set to monolayer collision steps. Figure 3 shows the trajectories of the first 500 helium ions in the sample. Very few of the ions were seen to backscatter to the surface of the sample. The yield of backscattered ions from the 10000 in the simulation was just $0.94 \%$. This result indicates that a slightly higher dose was received by the supported sample due to backscattering of the primary beam within the substrate. However the backscatter yield is so low that it alone cannot account for the significant difference in damage rate between the two samples that we have observed.

Another factor to consider is the removal of the substrate atoms from the sample by the primary beam; this process is known as sputtering. The sputtered ions can interact with the graphene on their way to the surface. The sputtering yield is defined as the number of sputtered atoms divided by the number of incident ions. From the SRIM simulation we know that the sputtering yield for graphene is 0.035 . The sputtering yield is 0.003 for $\mathrm{Si}$ and 0.005 for $\mathrm{O}$. These sputtered atoms have a very low energy when compared to that of the primary beam. They therefore have a shorter elastic mean free path (MFP) than the primary beam. The sputtered atoms will transfer more energy to the atomic nuclei than to the sample electrons [33]. These various collisions contribute to the enhanced rate of damage in the supported graphene sample over the freestanding sample. The sputtering yield for freestanding graphene is just 0.005 .

Four images of a few layer graphene flake acquired with various doses in the HIM are shown in figures 4(a)-(d). Three of these images were acquired with doses very similar to those investigated by Raman spectroscopy $\left(5 \times 10^{13}, 10^{14}\right.$, $5 \times 10^{14} \mathrm{He}^{+} \mathrm{cm}^{-2}$ ) and one with a much higher dose of
$1.3 \times 10^{17} \mathrm{He}^{+} \mathrm{cm}^{-2}$. An intensity profile from each image was plotted across the top edge of the flake as indicated in figures $4(\mathrm{a})-(\mathrm{d})$. These profiles are shown to the right of the image from which they were plotted. In the first half of each profile there is no signal, this indicates that the experimental set up has successfully minimized any signal generation from beneath the sample. As the beam then scans across the sample a signal is observed. In the lowest dose image (figure 4(a)) the signal to noise ratio is very poor and the sample edge is not well defined. This image is almost entirely composed of black and white pixels without any grey levels in between. The signal to noise is increased as the imaging dose increases, however it is not until a significant dose, on the order of $10^{17} \mathrm{He}^{+} \mathrm{cm}^{-2}$ (figure 4(d)), that a good signal to noise ratio is achieved. This dose is over 200 times the maximum dose which we have investigated by Raman spectroscopy. From the intensity profile of the image acquired at the highest dose an accurate edge profile measurement could be made. The graphene edge is assumed to be perfectly sharp; we then attribute the distance over which the intensity drops off at the edge to the probe size of the helium ion beam. Taking the distance from $25 \%$ intensity to $75 \%$ intensity gives us a FWHM of our probe of $0.5 \pm 0.1 \mathrm{~nm}$. This measurement was done using the ImageJ [34] software package. The signal to noise ratio of the edge profile measurement from the low dose images could be improved by averaging over the width of several pixels. This approach could only be used in the limited case of a perfectly straight edge. Also, the image would still not reveal any other useful information such as surface details.

These results show us that in order to acquire an image of graphene with sub-nanometre resolution in the HIM, a dose which is hundreds of times beyond that which causes serious damage to the graphene lattice is required. Either a high quality sample or a high quality image can be attained, but not both.

When accurate topographical and dimensional analysis is required the HIM provides very useful information when compared to electron microscopes. The three images of a single graphene flake acquired in three different microscopes are shown in figure 5. Figure 5(a) is the SEM in-lens image, (b) is the STEM-HAADF image, and (c) is the HIM image. The corresponding intensity profiles are plotted adjacent. Each of the imaging techniques displays the graphene flake as a bright region on a dark background.

From the intensity profiles it can be seen that the edges are not always well defined. In the SEM and STEM profiles the edges are somewhat rounded, making it difficult to identify the exact edge of the flake. However, in the HIM profile the edges are bright. This results in a small intensity increase at the edge. This peak makes analysing the dimensions of the flake trivial in the HIM by measuring the peak to peak distance. The well-defined edge contrast in HIM images extends to smaller features than in low $\mathrm{kV}$ SEM images. The peak to peak method used here is a simple dimensional metrology technique for measuring nanoscale features, and is only available down to sub-nanometre features in the HIM. This is due to the enhanced sensitivity of secondary electron yield on incidence angle in the HIM. 

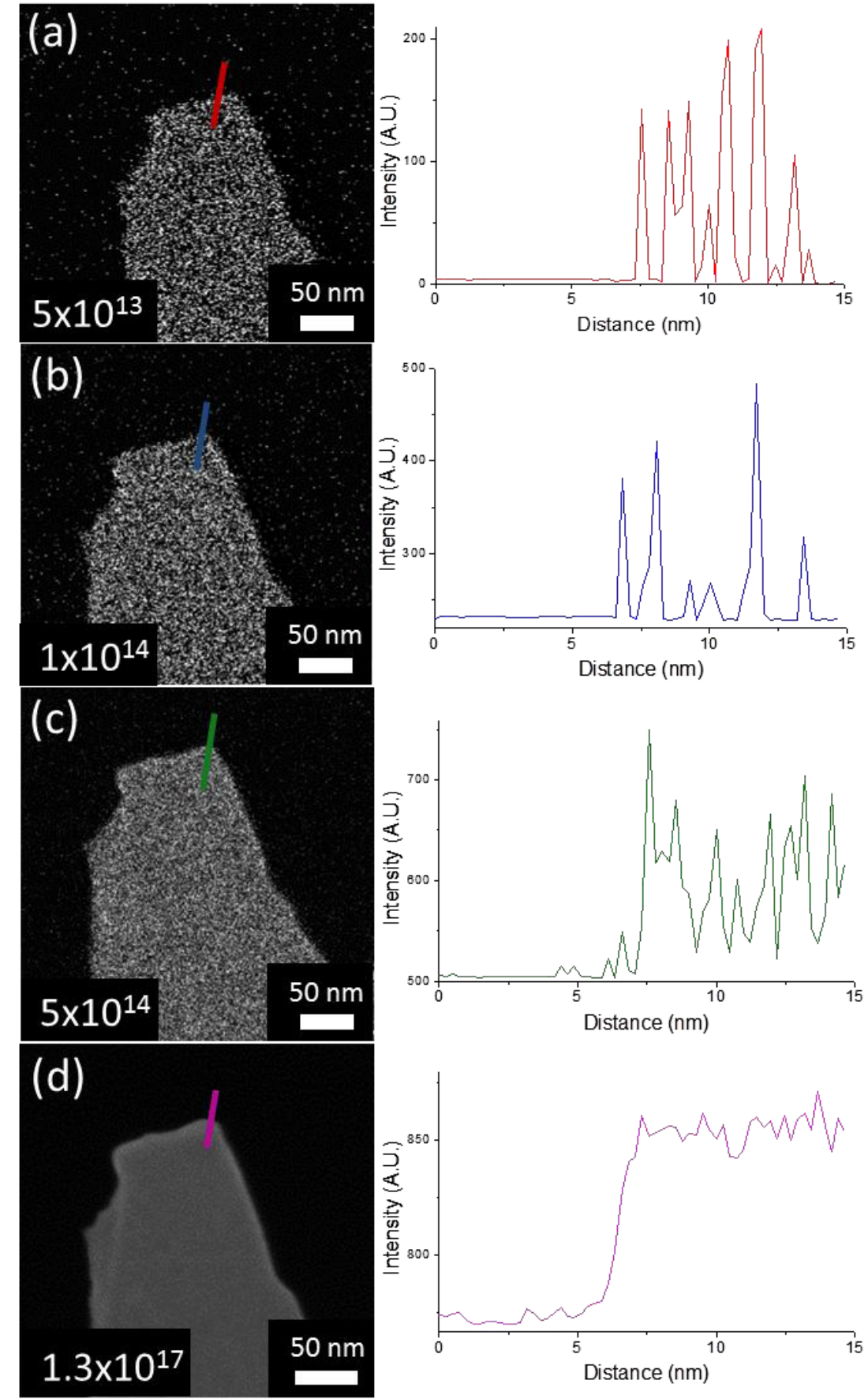

Figure 4. (a)-(d) Helium ion microscope images of a graphene flake acquired at various imaging doses. The flake is freestanding over a hole in a holey carbon support film. The imaging dose used is indicated on the image in units of $\mathrm{He}^{+} \mathrm{cm}^{-2}$. An intensity profile across the edge of the graphene flake was plotted from each image and is shown adjacent. A poor signal to noise ratio was observed until the highest dose of $1.3 \times 10^{17} \mathrm{He}^{+} \mathrm{cm}^{-2}$ was used. From the edge profile of the highest dose image the probe size was estimated to be $0.5 \mathrm{~nm} \pm 0.1 \mathrm{~nm}$.

\section{Conclusion}

In this work we have prepared graphene by both CVD and chemical exfoliation. Using the CVD graphene and Raman spectroscopy we determined that a dose of $30 \mathrm{kV}$ helium ions in the range of $10^{13} \mathrm{He}^{+} \mathrm{cm}^{-2}$ and below produces minimal damage to graphene. Doses above $5 \times 10^{14} \mathrm{He}^{+} \mathrm{cm}^{-2}$ cause significant damage to the graphene lattice. The presence of a substrate beneath the graphene was observed to enhance the rate of damage. The chemically exfoliated graphene flakes were then used to find the dose required to achieve sub-nanometre $(0.5 \mathrm{~nm})$ resolution in the HIM. A dose on the order of $10^{17} \mathrm{He}^{+} \mathrm{cm}^{-2}$ was required, 200 times greater than the dose which caused significant lattice damage in the Raman spectroscopy. A non-destructive image can be acquired in the HIM, but it must either have a lower signal to noise ratio or a lower magnification than our high dose image. 

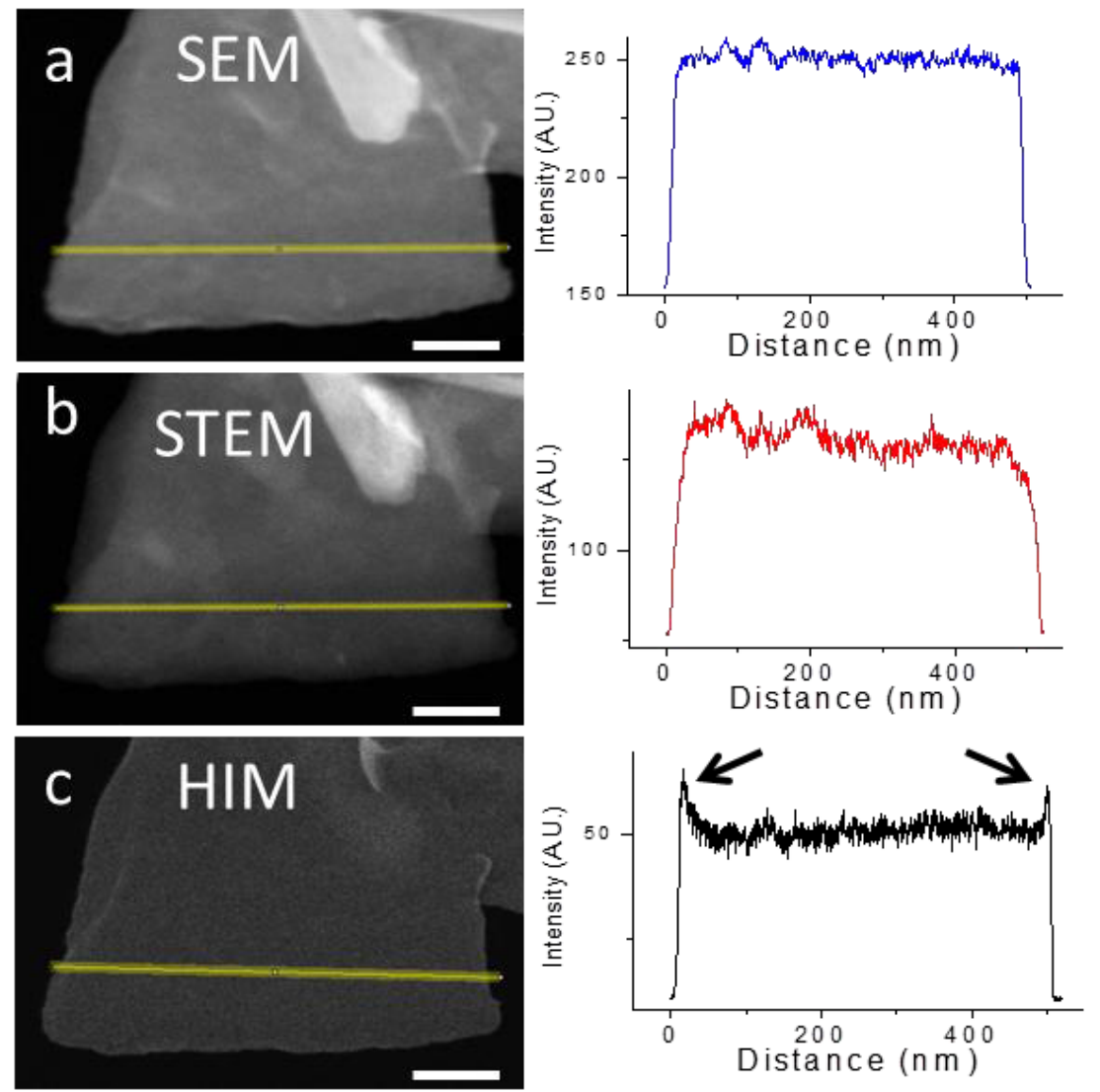

Figure 5. (a) SEM in-lens image of a graphene flake. An intensity profile across the flake is shown adjacent. (b) STEM-HAADF image of the same graphene. The intensity profile from the same region of the flake is shown adjacent. (c) HIM image of the same flake, with the intensity profile from the same region also plotted. The well-defined flake edges in the HIM intensity profile are indicated by two arrows.

HIM images of a single chemically exfoliated graphene flake were then compared with SEM and STEM images of the same flake. The edge of the flake is most clearly defined in the HIM image. Samples with nanometre or even sub-nanometre dimensions, down to single atom thickness, can be simply and accurately measured by helium ion microscopy.

The HIM provides highly precise material modification, both in terms of lateral dimensions, desired dose, and even penetration depth into the sample by adjusting the acceleration voltage. This modification has been used here to introduce defects into a graphene sample. While this modification is useful for tailoring material properties, its effect must also be considered when imaging the sample. Images may be acquired with relatively non-destructive imaging doses of $10^{13}-10^{14} \mathrm{He}^{+} \mathrm{cm}^{-2}$. However, when acquiring images with sub-nanometre resolution a significant amount of damage is introduced into the sample in order to acquire an image with a good signal to noise ratio. The doses identified here serve as a guideline for safely imaging, or controllably modifying graphene in the HIM.

\section{Acknowledgments}

We would like to thank Dr Alan Bell and Mr Clive Downing at the CRANN Advanced Microscopy Laboratory at Trinity College for their assistance and useful discussions. The work at the School of Physics and the Centre for Research on Adaptive Nanostructures and Nanodevices at Trinity College Dublin is supported by Science Foundation Ireland under Grants 11/PI/1105, 07/SK/I1220a and PI_10/IN.1/I3030. The TEM work was conducted under the framework of the INSPIRE program funded by the Irish Government's Program for Research in Third Level Institutions, Cycle 4, National Development Plan 2007-2013.

\section{References}

[1] Ward B, Notte J A and Economou N 2006 Helium ion microscope: a new tool for nanoscale microscopy and metrology J. Vac. Sci. Technol. B 24 2871-4

[2] Petrov Y V, Vyvenko O F and Bondarenko A S 2010 Scanning helium ion microscope: distribution of secondary electrons and ion channeling J. Surf. Investig. X-ray, Synchrotron and Neutron Tech. $4792-5$

[3] Ramachandra R, Griffin B and Joy D 2009 A model of secondary electron imaging in the helium ion scanning microscope Ultramicroscopy 109 748-57

[4] Postek M T and Vladár A E 2008 Helium ion microscopy and its application to nanotechnology and nanometrology Scanning 30 457-62

[5] Ishitani T, Yamanaka T, Inai K and Ohya K 2010 Secondary electron emission in scanning $\mathrm{Ga}$ ion, He ion and electron microscopes Vacuum 84 1018-24

[6] Inai K, Ohya K and Ishitani T 2007 Simulation study on image contrast and spatial resolution in helium ion microscope J. Electron Microsc. 56 163-9 
[7] Postek M T, Vladar A E and Ming B 2009 Recent progress in understanding the imaging and metrology using the helium ion microscope Proc. SPIE 7378737808

[8] Scipioni L 2008 Ultra-High Resolution Imaging in Orion Plus (Peabody, MA: Carl Zeiss)

[9] Scipioni L, Stern L A, Notte J, Sijbrandij S and Griffin B 2008 Helium ion microscope Adv. Mater. Process. 166 27-30

[10] Hlawacek G, Veligura V, Lorbek S, Mocking T F, George A, van Gastel R, Zandvliet H J W and Poelsema B 2012 Imaging ultra thin layers with helium ion microscopy: utilizing the channeling contrast mechanism Beilstein J. Nanotechnol. 3 507-12

[11] Bell D C 2009 Contrast mechanisms and image formation in helium ion microscopy Microsc. Microanal. 15 147-53

[12] Bell D 2011 Contrast performance: low voltage electrons versus helium ions Microsc. Microanal. 17 660-1

[13] Notte J, Farkas L, Scipioni L and Smith I 2010 Imaging of bio-compatible polymers in the helium ion microscope Microsc. Microanal. 16 200-1

[14] Ohya K, Yamanaka T, Inai K and Ishitani T 2009 Comparison of secondary electron emission in helium ion microscope with gallium ion and electron microscopes $\mathrm{Nucl}$. Instrum. Methods Phys. Res. B 267 584-9

[15] Postek M T, Vladár A, Archie C and Ming B 2011 Review of current progress in nanometrology with the helium ion microscope Meas. Sci. Technol. 22024004

[16] Novoselov K S, Geim A K, Morozov S V, Jiang D, Zhang Y, Dubonos S V, Grigorieva I V and Firsov A A 2004 Electric field effect in atomically thin carbon films Science 306 666-9

[17] Du X, Skachko I, Barker A and Andrei E Y 2008 Approaching ballistic transport in suspended graphene Nature Nanotechnol. 3 491-5

[18] Dean C, Young A, Meric I, Lee C, Wang L, Sorgenfrei S, Watanabe K, Taniguchi T, Kim P and Shepard K 2010 Boron nitride substrates for high-quality graphene electronics Nature Nanotechnol. 5 722-6

[19] Balandin A A, Ghosh S, Bao W, Calizo I, Teweldebrhan D, Miao F and Lau C N 2008 Superior thermal conductivity of single-layer graphene Nano Lett. 8 902-7

[20] Lee C, Wei X, Kysar J W and Hone J 2008 Measurement of the elastic properties and intrinsic strength of monolayer graphene Science $321385-8$

[21] Pickard D and Sciponi L 2009 Graphene Nano-ribbon Patterning in the Orion Plus (Peabody, MA: Carl Zeiss)
[22] Tapaszto L, Dobrik G, Lambin P and Biro L P 2008 Tailoring the atomic structure of graphene nanoribbons by scanning tunnelling microscope lithography Nature Nanotechnol. 3 397-401

[23] Fox D, Chen Y, Faulkner C C and Zhang H 2012 Nano-structuring, surface and bulk modification with a focused helium ion beam Beilstein J. Nanotechnol. 3 579-85

[24] Fox D, O'Neill A, Zhou D, Boese M, Coleman J N and Zhang H Z 2011 Nitrogen assisted etching of graphene layers in a scanning electron microscope Appl. Phys. Lett. 98243117

[25] Zhou Y B, Liao Z M, Wang Y F, Duesberg G S, Xu J, Fu Q, Wu X S and Yu D P 2010 Ion irradiation induced structural and electrical transition in graphene J. Chem. Phys. 133234703

[26] Kumar S, Rezvani E, Nicolosi V and Duesberg G S 2012 Graphene resist interlacing process for versatile fabrication of free-standing graphene Nanotechnology 23145302

[27] Fan J, Michalik J M, Casado L, Roddaro S, Ibarra M R and De Teresa J M 2011 Investigation of the influence on graphene by using electron-beam and photo-lithography Solid State Commun. 151 1574-8

[28] Peltekis N, Kumar S, McEvoy N, Lee K, Weidlich A and Duesberg G S 2012 The effect of downstream plasma treatments on graphene surfaces Carbon 50 395-403

[29] Cançado L G, Jorio A, Ferreira E H M, Stavale F, Achete C, Capaz R, Moutinho M, Lombardo A, Kulmala T and Ferrari A 2011 Quantifying defects in graphene via Raman spectroscopy at different excitation energies Nano Lett. $113190-6$

[30] O'Neill A, Khan U, Nirmalraj P N, Boland J and Coleman J N 2011 Graphene dispersion and exfoliation in low boiling point solvents J. Phys. Chem. C 115 5422-8

[31] Ferrari A C 2007 Raman spectroscopy of graphene and graphite: disorder, electron-phonon coupling, doping and nonadiabatic effects Solid State Commun. 143 47-57

[32] Ziegler J F, Biersack J P and Ziegler M D 2008 Stopping and Range of Ions in Matter (Chester, MD: SRIM Co.)

[33] Ramachandra R 2009 A study of helium ion induced secondary electron production PhD Diss. University of Tennessee

[34] ImageJ United States National Institutes of Health, [Accessed 02/02/2013], available from: http://rsb.info.nih.gov/ij/ 